\title{
Towards A Taxonomy of Autonomous Vehicle Handover Situations
}

\author{
Roderick McCall ${ }^{1}$ Fintan McGee ${ }^{1}$ Alexander Meschtscherjakov ${ }^{2}$ Nicolas Louveton, Thomas Engel ${ }^{3}$ \\ \{firstname.lastname\}@list.lu@ alexander.meschtscherjakov@sbg.ac.at \{firstname.lastname\}@uni.lu \\ ${ }^{1}$ eScience Group, Luxembourg Institute of Science and Technology (LIST), Luxembourg \\ ${ }^{2}$ Center for HCI, University of Salzburg, Salzburg, Austria \\ ${ }^{3}$ Interdisciplinary Centre for Security, Reliability and Trust, University of Luxembourg, Luxembourg
}

\begin{abstract}
This paper proposes a taxonomy of autonomous vehicle handover situations with a particular emphasis on situational awareness. It focuses on a number of research challenges such as: legal responsibility, the situational awareness level of the driver and the vehicle, the knowledge the vehicle must have of the driver's driving skills as well as the in-vehicle context. The taxonomy acts as a starting point for researchers and practitioners to frame the discussion on this complex problem.
\end{abstract}

\section{Author Keywords}

Autonomous vehicles; handover; transition; taxonomy.

\section{ACM Classification Keywords}

H.1.2 User/Machine Systems: Human Factors; H.5.2 User Interfaces: Evaluation / Methodology

\section{INTRODUCTION}

Autonomous vehicles are enjoying significant media attention and public awareness. A 2014 French research study found that the majority $(68.1 \%)$ of the public accepted the idea of fully automated driving [20]. Estimates regarding the delivery of fully autonomous cars range from three to thirty years [6]. Despite the excitement however problems remain, in particular with the reliability of the autonomous systems. In the case of Google's self -driving car (see Figure 2) the vast majority of crashes have been as a result of the human drivers in other vehicles [23], with the system first being responsible for a crash in 2016. This would suggest the need for such cars and their drivers to maintain full situational awareness of the environment around them and to maintain the degree of skills required to drive a vehicle. This brings to the fore the essential paradox of autonomous vehicles in that while driving skills will no-longer be required for the daily driving task they will become essential under extreme conditions. This will place significant

Permission to make digital or hard copies of all or part of this work for personal or classroom use is granted without fee provided that copies are not made or distributed for profit or commercial advantage and that copies bear this notice and the full citation on the first page. Copyrights for components of this work owned by others than the author(s) must be honored. Abstracting with credit is permitted. To copy otherwise, or republish, to post on servers or to redistribute to lists, requires prior specific permission and/or a fee. Request permissions from Permissions@acm.org. Automotive'UI 16, October 24 - 26, 2016, Ann Arbor, MI, USA Copyright is held by the owner/author(s). Publication rights licensed to ACM.

ACM978-1-4503-4533-0/16/10_..\$15.00

DOI: http://dx.doi.org/10.1145/3003715.3005456 demands on drivers who are unfamiliar with driving under potentially high cognitive load and complex situations. Related to this are the questions of responsibility and resumption of control.

The following paper presents a review of areas relating to autonomous vehicle handover situations. These are not intended to be an exhaustive list but a starting point to frame future discussions. The areas explored include: the in-car space, automation, situational awareness, reclaiming control, skills and driver profiling. A taxonomy is then presented along with a discussion, future challenges and a conclusion. The taxonomy explores not only the nature of situational awareness (SA) from the perspective of what the driver must possess but also what SA the car must possess and how this could be shared with the driver in the event of a handover situation. It also examines whether the vehicle should possess knowledge of the driver's skills.

\section{BACKGROUND}

\section{Responsibility}

The driving environment plays a significant role in the success of these cars. The project director for Google's autonomous car project has stated that "we are going to find places where the weather is good, where the roads are easy to drive - the technology might come there first"'[6]; while this is probably related to the testing phase of such vehicles it implies that certain contexts are currently, and perhaps also in the future, beyond the scope of autonomous vehicles. Extending this further it suggests that if not solved the issue of responsibility still rests with the driver. At least when they are driving "within an unfriendly context". In contrast Volvo has been at the forefront of accepting responsibility with its CEO Hakan Samuelsson stating: "Volvo will accept full liability whenever one of our cars is in autonomous mode. We are one of the first car makers in the world to make such a promise".

The problem of responsibility becomes more complex when viewed from the perspective of different types of handover situation (outlined later), for example if the car suddenly hands over control due to not being able process a particular context. Furthermore, should the vehicle be aware of the driver's skills and restrict how and when the driver can resume control? Therefore, while Volvo's promise to accept 
responsibility in autonomous mode is significant, it does not address all the relevant issues.

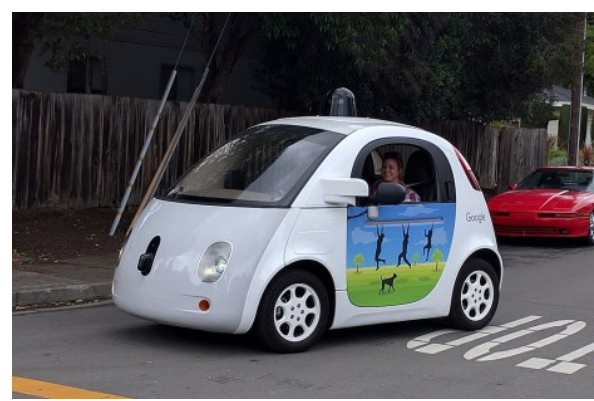

Figure 1 Google Self-Driving Car. Image: CC BY-SA 4.0 by Grendelkhan on Wikimedia. ${ }^{1}$

\section{Automation}

Vehicular automation can be defined in three levels: advisory, automatic or fully automatic [24]. The NHTSA provides more specific guidance in the form of levels 0 to 4 , with L0 representing no automation, L1 specific functions under control (e.g. brakes), L2 combined function automation (e.g. adaptive cruise control), while L3 provides limited self-driving (automation of all critical functions within certain contexts) and L4 (full automated control at all times).

The Google Car while aiming for L4 is in reality operating at L3, as (for now) it assumes friendly driving contexts. In both cases the level of automation fundamentally changes the relationship between the driver and the vehicle [25], to one where it could be argued the driver is little more than a passenger and where their role is to monitor the current driving context and (if possible within the chosen vehicle) respond when the need arises [25]. A more fine grained model of levels of automation (in the non-automotive context) has been presented by Parasuraman et al [19]. They distinguish between 10 levels of automation, from (1) The computer offers assistance, but the human decides and acts until (10) The computer acts autonomously, ignoring the human. Autonomous systems also have problems in that they can encourage operator complacency [34] and confusion can arise as to understanding what the autonomous systems is doing [27]- either users are under the impression it is doing nothing or that it is doing something else. As a result, there is a need to provide systems which keep the driver in the loop, reduce complacency and which provide a clear current status overview.

\section{The In-Car Space}

The nature of the in-car experience will change, and along with it the share of tasks and responsibilities that are split between the car and the driver. This is in part due to the nature of automation which will mean the weighting given to the three different driving tasks: primary, secondary and

\footnotetext{
${ }^{1}$ Google Self Driving Car by Grendelkhan on Wikimedia https://goo.gl/ohtq3P Licence: CC BY-SA 4.0

(retrieved 14-10-16)
}

tertiary will change. At the most basic level these map onto input devices. For example, the primary task of maneuvering the car (steering etc.) will be taken over by onboard systems, as most likely will many secondary tasks such as using windscreen wipers and indicators and perhaps some tertiary tasks such as adjusting heating etc. Increasingly the occupants of the vehicle will have more time on their hands to do something else (tertiary tasks) such as using entertainment system, relaxing or speaking to and interacting with others. This has led to a debate on the future in-car experience on autonomous vehicles (see workshops such as [17]).

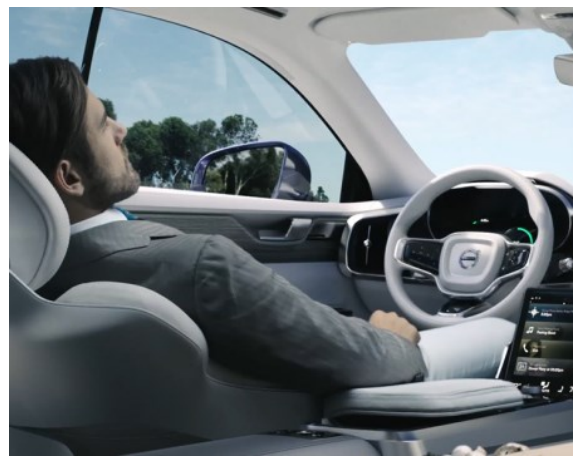

Figure 2 (c) Volvo Cars example of person relaxing.

Kerr [9] noted the phenomenon of DWAM (driving without attention mode). In this mode, normal drivers are unable to recall how they reached a destination due to predictability and prior experience of their route. It could be said that this is similar to the problem that drivers in autonomous and semi-autonomous vehicles may face as their lack of control of the vehicle is likely to encourage them to focus their attention elsewhere. Extending this further the driver in an autonomous vehicle will end up having a similar experience to that of the person who was traditionally thought of as a passenger. With this in mind it is not entirely unrealistic to assume (as some have suggested) that drivers may be able to read newspapers, relax (see Figure 2) or play games. This means that on a personal level the driver may perceive DWAM as desirable. Further along the spectrum of new experiences, Krome [10] suggests that undertaking fitness activities while not driving may be one option with meetings and playing games being other possible alternative activities.

Until now the in-car design space has remained relatively static[8]. However, as the in-car experience changes the need for to redesign will become more common. With Google for example doing away with the steering, while (for now) Volvo appear to be retaining it (see Figure 2). The change in the methods of control will also have a major impact on the driving task and it should be noted that Google are aiming 
for fully autonomous vehicles which should never require the driver to resume control.

\section{Situational Awareness}

If drivers are encouraged to undertake other tasks then there is a risk of a drop in situational awareness. A lack of situational awareness according to Gugerty [7] is the most likely cause of real time driving mistakes and it is noted that SA is likely to be higher when people have active control of a vehicle. Gugerty noted that passengers exhibit very dangerous patterns of situational awareness[7]. If these patterns were repeated within a (semi-)autonomous driving context the "driver" would not have sufficient information in order to take a safe course of action when responding to an event.

\section{Driver}

In order for a driver to be able to act appropriately in response to an event they must possess situational awareness. SA as defined by Endsley [4] consists of three levels:

- L1: ability to perceive elements within an environment, - L2: comprehend their meaning in relation to task goals

- L3: projection in to the future after particular action(s).

Broadly speaking these can also be mapped onto levels of driving tasks [33] [15], which are: operational, tactical and strategic. Operational tasks relate to low-level manual control (e.g. pressing a pedal) and are often semi-automated by the driver and require L1 situational awareness. Tactical tasks, for example maneuvering a car in traffic, require more complex control use and awareness and therefore require L1 and L2 situational awareness. However, they require a relatively short projection into the future - meaning the ability to predict the outcome of events. Finally, strategic level driving tasks reflect longer-term navigation plans and require all three levels of situational awareness. In addition to the levels of SA defined above Matthews [15] defines further components:

- Spatial awareness (location relative to the environment)

- Identity awareness (knowledge of salient items)

- Temporal Awareness (changes over time)

- Goal Awareness (status relevant to current goals)

- System Awareness (awareness of the car systems).

In the UK, advanced drivers are assumed to operate at a level above merely responding to the current situation [32]. They are deemed to be able to look for patterns, chunk this knowledge and apply it across different situations. This would imply L1-L3 situational awareness from the Endsley perspective, and would also combine the aspects described by Matthews, including aspects such as temporal (projection into the future) and goal awareness.

\section{Workload}

With (semi-)autonomous vehicles offering drivers the chance to do something else, driver distraction also becomes and problem. For example, [12] noted that the mental workload increase caused by using a cell phone with active cruise control will decrease SA.

\section{Vehicle}

While the driver is required to maintain situational awareness, vehicles also increasingly automate this task. For example, (semi-)autonomous vehicles have a range of technologies such as sensors, cameras and eye trackers coupled with telemetry data which allow them to be aware of the current state of the vehicle relative to the environment. This form of automated situational awareness is critical in handover situations, as it could be used to assist the driver in decision making even if the vehicle itself is not in control e.g. via visualizations of routes or dangers etc. Therefore, how and when such information is provided becomes important.

\section{Skills}

Skills are retained in two primary forms: declarative and procedural knowledge. Declarative knowledge involves conscious control; this would be similar to when someone is learning to drive. In this context they are very aware of the pedals, steering wheel and lateral positioning of the vehicle. In contrast procedural knowledge is automated, fast and requires little attention. Normally, skills are acquired first declaratively then become encoded procedurally and successful completion requires one or a mix of both approaches.

Research on skills acquisition suggests that declarative knowledge has a lower retention rate over time than procedural knowledge [22]. Which is a positive indication for drivers as it has been anecdotally suggested that driving car is not a skill which is easily forgotten; rather like riding a bike. However, it has been noted that in general skills must be practiced frequently in order for them to be retained. Within an automated driving context this becomes problematic as driver's will have less day to day exposure to the task of driving, yet in handover situations the right skills are essential. It is further compounded by research which suggests that driving performance degrades as the level of vehicle automation increases [28].

\section{Driver Skills Profiling}

Safely transitioning from automated to manual driving is a challenging problem, one which requires knowledge of the driver's skills. The skills which drivers possess not only impact on their performance under normal and resumption of control situations but are also relevant to how, when and under what conditions the car could hand back control under non-emergency situations. In addition, while raw skills should be assessed there is also need to assess how a particular driver performs under different circumstances, for example when under a high cognitive load environment (e.g. using a mobile phone, talking and having to resume control). As an example of the benefit of such approaches, Nilsson et al. [18] have developed an approach to allowing safe transition based on characterizing the drivers capabilities, as they state that understanding a drivers capabilities is necessary for a safe transition. The time taken for transition 
has also been shown to be a factor, as when transition time is shorter, while decision making and reactions are faster, the quality is lower [5]. Given the benefits of understanding the driver's skill level, it therefore becomes important to look at which approaches are available.

The increasing use of black box type driver profiling technologies allows for the relatively easy collection and storage of raw car telemetry data. These type of devices allow for collection of rich data and for post-processing e.g.: overlaying GPS routes with the road network to determine adherence to local speed limits. These technologies are gaining popularity within the UK insurance sector while they can be useful for assessing raw quantitative information e.g. speed etc. However, the interpretation of these statistics with respect to assessing more complex driving skills is on-going work and open to debate from a scientific, ethical and legal perspective. Participants in experiments have shown a preference for black box type data collection approaches over self-reported data diary methods [14] as they do not require manual data entry (as in the case of diaries).

While black box car telemetry data systems allows for the collection and possible interpretation of raw data, other approaches are also available such as questionnaires [29], evaluation by observers [13], analysis of historical data, and the use of simulators. At face value the more subjective approaches may seem out of scope for autonomous vehicles, especially given their relatively basic technical approaches. They are also not without limitations. However, studies using questionnaires have shown drivers think that they are better than the average driver [29] but it is quite unlikely that this is the case. In contrast the use of historical data is less likely to be effected by bias or over estimation [29]. Simulators and observer based approaches while useful for laboratory based trials and controlled situations would not be practical for regular testing of drivers on a large scale.

While questionnaires and black box data approaches are relevant, the former would require regular assessment of the driver which may result in low response rates or bad data as drivers may become frustrated with endless data collection. Black box approaches would appear to be more relevant. However, the paradox here is that automated vehicles will reduce the scope for driver data collection. Therefore, methods must be found to encourage drivers to (a) drive more frequently than required and/or (b) encourage regular self-reported assessment of their perceived skills.

\section{DEVELOPING A TAXONOMY}

In this section we begin developing a taxonomy of handover situations and the required attributes or intrinsic properties (see Table 1 Taxonomy of Handover Contexts). We begin by exploring the current thinking regarding handover situations then define five forms of handover situation. Following from this we explore each hand over situation with respect to responsibility, situational awareness level, driver required skill level and the level of driver profiling knowledge required.

\section{Handover Situations}

Shaikh and Krishnan [26] defined three main problems with semi-autonomous vehicles: handover situations, inadequate feedback and a fundamental change in the driving task. Furthermore, previous work by Walch et al. [31] distinguishes between immediate handover (e.g., when drivers grasp the steering wheel), a stepwise handover Control (e.g. first longitudinal control followed by lateral control, or vice versa), driver monitored Handover (e.g., by grasping the steering wheel and after a countdown, the control is handed over), and system monitored handover (e.g., the system monitors the inputs of the driver after the handover). These handovers are described from the perspective of procedure and are characterized by the division of control between the driver and the system. Our focus is on the context of the handover, specifically with respect to the situational awareness of the driver. Here we propose five forms of handover that are from the perspective of the vehicle handing control to the driver. It should be noted that handovers can also occur in the other direction. For example, when a context is deemed safe enough the vehicle could indicate that it can resume control and let the driver decide whether to permit this. However, when this is the case it can be reasonably assumed that the driver already possess some degree situational awareness as he or she is driving.

In general, hand over situations can be divided into those which are scheduled (i.e. it is known in advance that there will be a hand-over at a certain point in time) and nonscheduled (i.e. the hand-over is not planned in advance but occurs non-scheduled) hand-over situations. If a hand-over situation occurs non-scheduled we can distinguish between system or user initiated hand-overs. Finally, we can add timing as a crucial factor in the hand-over situation. This leaves us with five forms of hand-over situation as detailed below:

\section{Scheduled}

An example scenario is when a car is entering an area not suitable for automated driving. The driver will be notified in advance and at a suitable point will be asked to resume control. Probably, the driver has to actively indicate to take over control (e.g., by pulling two handles). The driver should be made aware of the driving context so that they can prepare to take over control. An example would be if the system provided the driver with a warning $1 \mathrm{~km}$ before they arrived in a complex environment then hands over control with appropriate warnings. The vehicle has to assume that the driver has sufficient skills and situational awareness to take on this task therefore with appropriate route planning software the system should handover only when appropriate.

\section{Non-scheduled system initiated}

This would be a case where system realizes that the driver must take control, perhaps due to a sudden change in road conditions, as the system would need to operate beyond its functional limits (this is referred to as self-deactivation by [18]). The driver may not have been expecting this and it will 
be necessary for the system to ensure that the driver has complete awareness of the driving environment. At this point the vehicle, while perhaps being aware of driver skills, has to handover control regardless.

\section{Non-scheduled user initiated}

This is when the driver decides to take control when there is no specific need for them to do so. An example would be if the driver wishes to resume control in order to experience the thrill of driving. Another example could be that the driver wants to take a different route and is not able to communicate this to the vehicle (e.g., follow a specific vehicle in front). In this scenario the driver could use the same interface (e.g. two pull handles) to take control as used in scheduled handover situations. As automated vehicles become more advanced one critical aspect emerges, that is whether the car should relinquish control if it suspects the driver will not have the required skills?

\section{Non-scheduled user initiated emergency}

In this case the user has spotted a potential catastrophic risk the system has failed to see and takes immediate control. At this point there might not be the time for the driver to initiate a handover procedure e.g., by pulling two handles. It could be the case that the driver intuitively grasps the steering wheel and turns it round. A primary concern is how the autonomous vehicle should react. Should the driver always have the right to overrule the vehicle? What if the vehicle would hurt somebody if it follows the driver's commands? What if the driver unintentionally took over control? In terms of situational awareness the driver would seem to have a better understanding of the situation than the vehicle otherwise the driver would not have taken over. Another question is how to distinguish this from the non-emergency hand-over type.

\section{Non-scheduled system initiated emergency}

In this case the fault entirely lies within the system, and it is a case of internal system failure rather than an external threat. In this type of emergency the system can no longer function, and assuming it is possible to do so, the driver is notified. In this scenario it is often assumed that the vehicle at least has the ability to stop itself in a safe manner if the driver cannot or is not willing to take over control. In terms of situation awareness this might be the most crucial case, since time plays an important role and the system has to decide if the driver is capable of taking over control in a limited amount of time or if an emergency stop procedure is undertaken.

This scenario is also considered under the category selfdeactivation by [18] but we consider it a separate scenario as the response may be different. As a system approaches it's functional limits preparations can be made for a handover, in the case of a catastrophic internal failure bringing the system offline the handover must be immediate.

\section{Handover Event Timeline and Return to Automation}

The handover situation is split-up into two phases, the first alert and the event itself. This reflects the key difference between scheduled and non-scheduled events. For example, when a scheduled handover event occurs the driver and vehicle have a period of time before the actual event takes place. In contrast when non-scheduled events occur there will most likely be no time between an alert and the event occurring. In order to reflect this the following handover event timeline is used:

1. First alert of an event (referred to as first alert). This is when the driver is first informed they have to take control or when they first indicate they will take control.

2. Resumption of control during the event by the driver (referred to as the event)

3. Return to automated control once vehicle can resume control or the driver assigns control. (referred to as hand back).

The assumption here is that the vehicle will not take back control unless it knows it can undertake the maneuver safely.

\section{Situational Awareness}

This category indicates the level of knowledge that the driver must have in order to respond to the event and is based on the L1 to L3 model listed earlier. In the case of the taxonomy this is defined from the perspective of the degree of situational awareness required by the driver at the point of the first alert (e.g. the message) and during the handover event. SA can also be extended to the vehicle itself. In the taxonomy this is first presented as the SA knowledge the vehicle has at the time of the initial alert and also during the actual event. The event property is the relevancy of providing SA information during the event with respect to the current handover type. The levels are: critical, useful and N/A. In the case of non-scheduled events these are assumed not to have a first alert, rather the event occurs. Therefore, no SA level is provided for the first alert phase.

\section{Responsibility}

For responsibility level we assign a rating on the basis of which party should be responsible for the result of the handover situation, this is provided with a rating of 1: Driver Only, 2: Driver and partially the manufacturer 3: Driver and Manufacturer 4: Manufacturer and partially the Driver and 5: Manufacturer only. For those levels of autonomy where the driver is required to monitor the environment (level 0-2), the driver has at least some partial responsibility, whereas for the level in which the driver might not even be able to take over control (level 4) (for example, if the car does not have a steering wheel or pedals at all) most of the responsibility will rest on the manufacturer. It is at level 3 that the responsibility between the driver and the manufacturer seems to be blurred.

\section{Awareness of in-Car Context}

The vehicle may also have to be aware of current driving context and the interaction between the occupants, for example what they are currently doing and how to effectively communicate with the driver. For example, if during an event the car itself is aware that the driver is talking to someone it should perhaps increase the alert volume and/or use visual 
cues. In serious non-scheduled system initiated time critical handovers the system would use the highest level of alert, so it would not be applicable. corridor approach resulted in more consistent driver trajectories.

While auditory notifications are widely used and accepted in areas such as mobile phone use, a recently study with respect

Table 1 Taxonomy of Handover Contexts.

\begin{tabular}{|c|c|c|c|c|c|c|c|}
\hline $\begin{array}{l}\text { Handover } \\
\text { Situation }\end{array}$ & Responsibility & $\begin{array}{l}\text { Situational } \\
\text { Awareness. } \\
\text { Driver. } \\
\text { (First Alert) }\end{array}$ & $\begin{array}{l}\text { Situational } \\
\text { Awareness. } \\
\text { Driver. (Event) }\end{array}$ & $\begin{array}{l}\text { Situational } \\
\text { Awareness. } \\
\text { Vehicle } \\
\text { (First Alert) }\end{array}$ & $\begin{array}{l}\text { Situational } \\
\text { Awareness. } \\
\text { Vehicle } \\
\text { (Event) }\end{array}$ & $\begin{array}{l}\text { Vehicular System } \\
\text { Knowledge of } \\
\text { Driver Skills }\end{array}$ & $\begin{array}{l}\text { Awareness of } \\
\text { In-Car Context }\end{array}$ \\
\hline Scheduled & 1 & 3 & 3 & 3 & Useful & Required & Beneficial \\
\hline $\begin{array}{l}\text { Non-scheduled } \\
\text { system initiated }\end{array}$ & 4 & - & 3 & 3 & Useful & Beneficial & Beneficial \\
\hline $\begin{array}{l}\text { Non-scheduled user } \\
\text { initiated }\end{array}$ & 1 & 3 & 3 & 3 & Useful & Required & Beneficial \\
\hline $\begin{array}{l}\text { Non-scheduled user } \\
\text { initiated emergency }\end{array}$ & 4 & 3 & 3 & 3 & Critical & $\mathrm{N} / \mathrm{A}$ & $\mathrm{N} / \mathrm{A}$ \\
\hline $\begin{array}{l}\text { Non-scheduled } \\
\text { system initiated } \\
\text { emergency }\end{array}$ & 4 & - & 3 & 3 & Critical & $\mathrm{N} / \mathrm{A}$ & $\mathrm{N} / \mathrm{A}$ \\
\hline
\end{tabular}

\section{Knowledge of Driver's Skills}

In some cases, for example an emergency when a vehicle must hand over control knowledge of driver's skills is irrelevant. However, there may be situations where handing over control is undesirable and not handing over control would be safer. Therefore, if the car had knowledge of the driver's ability it may be able to suggest that the driver does not take over control or perhaps even prevent this altogether. This would raise additional ethical implications.

\section{FUTURE WORK}

\section{Handover Interfaces}

The paper has briefly touched on the problems of how to provide situational awareness information, however a detailed presentation of concepts is out of scope in this paper. That aside some possible approaches appear relevant including Data visualization. Data visualization has been used in a number of domains in order to help reduce complexity of large data sets or to present live time series data more effectively. Augmented Reality (AR) has already shown potential as a visualization technique for providing drivers with information $[3,30]$. However care needs to be taken that such visualization does not cause cognitive overload [16] and / or distract the driver. The choice of visual encoding of the information can have a large impact. For example Lorentz et al [11] evaluated two different approaches using augmented reality to aid drivers during takeover. In one approach augmented reality was used to visualize a green corridor that the driver could safely steer through, in the other the driver was shown a red corridor to be avoided. The authors found that, while the different types of visualization resulted in similar takeover times, the green to fully automated driving found that they were not viewed positively and are only tolerated [1]. Use of a 3D auditory displays was shown in a study to allow participants to acquire targets faster, although with no increase in accuracy [2]. Time is also a critical aspect within emergency handover situations.

Politis et al. [21] have utilized multimodal language-based warnings for the handovers of control in autonomous cars. They varied urgency and combinations of audio, tactile and visual warnings for handovers. Their results show that the recognition of the warning urgency is crucial for a successful handover. They observed quicker transitions for highly urgent warnings and poor driving performance for unimodal visual warnings.

\section{Ethical Issues}

As vehicles possess more situational awareness and potentially knowledge of driver skills it becomes important to consider if they should allow the driver to resume control under scheduled handover conditions? This has implications for the design of the vehicle, the rights of the driver and the responsibility of the manufacturer.

\section{Retaining Skills}

Retention of driving skills within the field of autonomous vehicles is an emerging area of research with to date one known project exploring it. A number of key areas that need to be addressed are: how to monitor skills and how to persuade people to maintain their skills.

\section{Taxonomy Development}

The proposed taxonomy represents only a starting point and does not include a more complex analysis of many issues, for example how skills will be retained as autonomous vehicles 
become more popular or how persuasive systems may be utilized to encouraged skills retention. Furthermore, it only focusses on certain aspects which were identified so far during our research. We acknowledge that many other aspects could be included.

\section{CONCLUSION}

This paper has presented a review of certain key areas related to situational awareness in the era of autonomous vehicles. Based on this review it has proposed an early taxonomy which places at its core three main areas: driver skills, situational awareness and different handover situations. In addition, it has started to explore the relevancy of responsibility in different situations and the effect of the incar context. It has also discussed ethical issues. The taxonomy will be useful in framing the discussion on autonomous vehicles in particular when it comes to identifying future areas of research.

\section{ACKNOWLEDGEMENTS}

The financial support by the Austrian Science Fund (FWF): I 2126-N15 and the Fonds National de La Recherche, Luxembourg under grant number: CS14/IS/8301419 are acknowledged. The authors acknowledge the assistance of the other members of the MADSAV project consortium.

\section{REFERENCES}

1. Pavlo Bazilinskyy and Joost de Winter. 2015. Auditory interfaces in automated driving: an international survey. PeerJournal of Computer Science 1: e13. http://doi.org/10.7717/peerj-cs.13

2. Durand R. Begault. 1993. Head-Up Auditory Displays for Traffic Collision Avoidance System Advisories: A Preliminary Investigation. Human Factors 35, 4: 707-717. http://doi.org/10.1177/001872089303500409

3. Daniel Damböck, Thomas Weißgerber, Martin Kienle, and Klaus Bengler. 2015. Evaluation of a contact analogue head-up display for highly automated driving. $4^{\text {th }}$ International Conference on Applied Human Factors and Ergonomics. San Francsico. USA

4. M.R. Endsley. 1988. Situation awareness global assessment technique (SAGAT). Proceedings of the IEEE 1988 National Aerospace and Electronics Conference, 789-795. http://doi.org/10.1109/NAECON.1988.195097

5. Christian Gold, Daniel Damböck, L. Lorenz, and K. Bengler. 2013. "Take over!” How long does it take to get the driver back into the loop? Proc. Human Factors and Ergonomics Society Annual Meeting 57, 1: 1938-1942.

http://doi.org/10.1177/1541931213571433

6. Lee Gomes. 2016. When Will Google's SelfDriving Car Really Be Ready? Three Super-Cool Technologies. IEEE Spectrum 53, 13-14.
7. Leo J. Gugerty. 1997. Situation awareness during driving: Explicit and implicit knowledge in dynamic spatial memory. Journal of Experimental Psychology: Applied 3, 1: 42-66. http://doi.org/10.1037/1076-898X.3.1.42

8. D Kern and A Schmidt. 2009. Design space for driver-based automotive user interfaces.

Proceedings of the 1st International Conference on Automotive User Interfaces and Interactive Vehicular Applications, 3-10.

9. John S Kerr. 1991. Driving without attention mode (DWAM): A formalisation of inattentive states in driving. In Vision in Vehicles - III. 473-479.

10. Sven Krome, William Goddard, Stefan Greuter, Steffen P. Walz, and Ansgar Gerlicher. 2015. A context-based design process for future use cases of autonomous driving. Proceedings of the 7 th International Conference on Automotive User Interfaces and Interactive Vehicular Applications AutomotiveUI '15, ACM Press, 265-272. http://doi.org/10.1145/2799250.2799257

11. L. Lorenz, P. Kerschbaum, and J. Schumann. 2014. Designing take over scenarios for automated driving: How does augmented reality support the driver to get back into the loop? Proceedings of the Human Factors and Ergonomics Society Annual Meeting 58, 1: 1681-1685. http://doi.org/10.1177/1541931214581351

12. Ruiqi Ma and David B. Kaber. 2005. Situation awareness and workload in driving while using adaptive cruise control and a cell phone.

International Journal of Industrial Ergonomics 35, 10: 939-953.

http://doi.org/10.1016/j.ergon.2005.04.002

13. Richard A. Marottoli and Emily D. Richardson. 1998. Confidence in, and self-rating of, driving ability among older drivers. Accident Analysis and Prevention 30, 3: 331-336. http://doi.org/10.1016/S0001-4575(97)00100-0

14. Shawn C Marshall, Keith G Wilson, Frank J Molnar, Malcolm Man-Son-Hing, Ian Stiell, and Michelle M Porter. 2007. Measurement of driving patterns of older adults using data logging devices with and without global positioning system capability. Traffic injury prevention 8, 3: 260-266. http://doi.org/10.1080/15389580701281792

15. Micheal L Matthews, David J Bryant, Robert D G Webb, and Joanne L Harbluk. 2001. Model for Situation Awareness and Driving: Application to Analysis and Research for Intelligent Transportation Systems. Transportation Research Record 1779, 26-32. 
16. Richard E. Mayer. 2005. The Cambridge handbook of multimedia learning. Camburdge University Press. http://doi.org/10.1075/idj.16.1.13pel

17. Alexander Meschtscherjakov, Manfred Tscheligi, Dalila Szostak, et al. Workshop on User Experience of Autonomous Driving. Adjunct Proceedings of the 5th International Conference on Automotive User Interfaces and Interactive Vehicular Applications (AutomotiveUI'13): 71-73.

18. Josef Nilsson, Paolo Falcone, and Jonny Vinter. 2014. Safe Transitions From Automated to Manual Driving Using Driver Controllability Estimation. IEEE Transactions on Intelligent Transportation Systems 16, 4: 1806-1816. http://doi.org/10.1109/TITS.2014.2376877

19. R. Parasuraman, T.B. Sheridan, and C.D. Wickens. 2000. A model for types and levels of human interaction with automation. IEEE Transactions on Systems, Man, and Cybernetics - Part A: Systems and Humans 30, 3: 286-297. http://doi.org/10.1109/3468.844354

20. William Payre, Julien Cestac, and Patricia Delhomme. 2014. Intention to use a fully automated car: Attitudes and a priori acceptability.

Transportation Research Part F: Traffic Psychology and Behaviour 27, PB: 252-263. http://doi.org/10.1016/j.trf.2014.04.009

21. Ioannis Politis, Stephen Brewster, and Frank Pollick. 2015. Language-based multimodal displays for the handover of control in autonomous cars. Proceedings of the 7th International Conference on Automotive User Interfaces and Interactive Vehicular Applications (AutomotiveUI '15): 3-10. http://doi.org/10.1145/2799250.2799262

22. Michael I. Posner. 1973. Cognition: An Introduction. Oxford.

23. Matt Richtel and Conor Dougherty. 2015. Google's Driverless Cars Run Into Problem : Cars With Drivers. The New York Times. https://goo.gl/UiHakx (retrieved: 12-10-2016)

24. Rosengren. 1995. Driver assistance and cooperative driving. ERTICO, First World Congress on Advanced Transport Telematics and Intelligent Vehilce Highway Systems: 1613-22.

25. N B Sarter, D D Woods, and C E Billings. 1997. Automation Surprises. In, Handbook of Human Factors \& Ergonomics. G Salvendy (Ed.). Wiley.

26. Siraj Shaikh and Padmanabhan Krishnan. 2012. A Framework for Analysing Driver Interactions with Semi-Autonomous Vehicles. Electronic Proceedings in Theoretical Computer Science 105: 85-99. http://doi.org/10.4204/EPTCS.105.7
27. Neville A. Stanton and Philip Marsden. 1996. From fly-by-wire to drive-by-wire: Safety implications of automation in vehicles. Safety Science 24, 1: 35-49. http://doi.org/10.1016/S0925-7535(96)00067-7

28. Niklas Strand, Josef Nilsson, I. C MariAnne Karlsson, and Lena Nilsson. 2014. Semi-automated versus highly automated driving in critical situations caused by automation failures. Transportation Research Part F: Traffic Psychology and Behaviour 27, PB: 218-228. http://doi.org/10.1016/j.trf.2014.04.005

29. Anna Sundström. 2008. Self-assessment of driving skill - A review from a measurement perspective.

Transportation Research Part F: Traffic

Psychology and Behaviour 11, 1: 1-9. http://doi.org/10.1016/j.trf.2007.05.002

30. M Tonnis, C Sandor, G Klinker, C Lange, and H Bubb. 2005. Experimental evaluation of an augmented reality visualization for directing a car driver's attention. Mixed and Augmented Reality, 2005. Proceedings of the Fourth IEEE and ACM International Symposium on, 56-59.

31. Marcel Walch, Kristin Lange, Martin Baumann, and Michael Weber. 2015. Autonomous Driving: investigating the feasibility of car-driver handover assistance. Proceedings of the 7th International Conference on Automotive User Interfaces and Interactive Vehicular Applications (AutomotiveUI'15): 11-18. http://doi.org/10.1145/2799250.2799268

32. Guy H. Walker, Neville A. Stanton, Tara A. Kazi, Paul M. Salmon, and Daniel P. Jenkins. 2009. Does advanced driver training improve situational awareness? Applied Ergonomics 40, 4: 678-687. http://doi.org/10.1016/j.apergo.2008.06.002

33. Nicholas J. Ward. 2000. Automation of task processes: An example of intelligent transportation systems. Human Factors and Ergonomics in Manufacturing 10, 4: 395-408. http://doi.org/10.1002/15206564(200023)10:4<395::AID-HFM3>3.0.CO;2-3

34. E. L. Wiener and R. E. Curry. 1980. Flight-deck automation: promises and problems. Ergonomics 23, 10: 995-1011. http://doi.org/10.1080/00140138008924809 